\title{
Big equation for small kidneys: a newly proposed model to estimate neonatal GFR
}

\author{
Abby Basalely ${ }^{1} \cdot$ Diane Liu $^{1} \cdot$ Frederick J. Kaskel ${ }^{1}$ (D)
}

Received: 24 June 2019 / Revised: 13 December 2019 / Accepted: 18 December 2019 / Published online: 31 January 2020

(C) IPNA 2019

\section{Background}

Rapid assessment of glomerular filtration rates (GFR) in infants is imperative for both drug dosing and recognition of acute kidney injury (AKI) [1-3]. This is especially important in low birth weight and preterm neonates who have an increased risk of AKI and development of chronic kidney disease (CKD) [4]. However, obtaining accurate estimates of GFR in term and preterm infants has been challenging. Serum creatinine, the traditional biomarker of filtration, is dependent upon muscle mass and age-dependent renal excretion. Creatinine values in newborns are further complicated by the fact that infant creatinine levels are reflective of maternal creatinine levels in the first few days of life and creatinine is reabsorbed by immature tubules $[5,6]$.

Traditional urinary clearances using the gold standard marker inulin have been used in the seventies to provide the basic information on the development of glomerular filtration rate $[7,8]$. GFR is low at birth with a value close to $20 \mathrm{ml} / \mathrm{min}$ per $1.73 \mathrm{~m}^{2}$ in term neonates. It is lower in premature infants. GFR develops rapidly after birth, its value doubling within the first 2 weeks of life. It develops at a somewhat lower velocity in very premature infants [9].

Recent studies by Vieux et al. [10] have assessed the urinary clearance of creatinine during the first 28 days of life of premature infants with various gestational ages, ranging from 27 to 31 weeks. They provided regression lines for estimating the expected value normal creatinine clearance at different gestational and postnatal ages. The results of these studies

Abby Basalely and Diane Liu contributed equally to the preparation of this manuscript. $\mathrm{AB}$ and DL are trainees on an NIH T32 NIDDK 5T32DK007110- 46.

Frederick J. Kaskel

fkaskel@montefiore.org

1 Department of Pediatrics, Division of Pediatric Nephrology, The Children's Hospital at Montefiore Bronx, 3326 Bainbridge Avenue, Bronx, NY 10467, USA were in agreement with the reference values established previously using inulin as a marker $[7,8]$.

However, estimating GFR by the traditional urinary clearance has an important drawback: it requires precisely timed collections of urine. For these reasons, methods without the need for urine have been sought after.

In 1976, Schwartz et al. published the first method of noninvasive estimation of GFR in children [11]. The researchers created a model to calculate eGFR in children 6 months18 years old, challenging the original estimation based on adults:

Creatinine Clearance $=\frac{\mathrm{UCr} \times \mathrm{V}}{\mathrm{SCr}}$

$\mathrm{UCr}$ is urinary creatinine $(\mathrm{mg} / \mathrm{dL}), \mathrm{SCr}$ serum creatinine $(\mathrm{mg} / \mathrm{dL})$, and $\mathrm{V}$ urinary flow rate $(\mathrm{mL} / \mathrm{min})$.

Schwartz et al. maintained that utilization of a ratio dependent on serum creatinine production did not take into account muscle mass, which varies in growing children. They performed a multivariate linear regression modeling of GFR initially measured by 24 -h creatinine clearance studies demonstrating that the ratio of height in centimeters $(\mathrm{cm})$ to $\mathrm{SCr}$ was the most significant predictor of GFR. Height was multiplied by the constant of $0.55(\mathrm{~L} / \mathrm{SCr})$, the coefficient derived from multivariate linear regression:

eGFR $\mathrm{ml} / \mathrm{min} / \mathrm{m}^{2}=\frac{0.55 \times \text { height }(\mathrm{cm})}{\mathrm{SCr}(\mathrm{mg} / \mathrm{dL})}$

They subsequently validated their model directly measuring GFR via single-injection inulin clearance in 77 of the pediatric patients.

In 1984, the group sought to develop an estimation of GFR in term infants [12]. They evaluated creatinine clearance in 137 infants aged 5 days -1 year, for which clearance was measured directly by single-injection inulin in 63 infants. The same relationship of height in $\mathrm{cm} / \mathrm{SCr}$ was substantiated, but the coefficient of 0.55 grossly overestimated GFR. Based on models built specifically for that population, a new constant of 
0.44 was developed for term neonates.

eGFR $\mathrm{ml} / \mathrm{min} / \mathrm{m}^{2}=\frac{0.44 \times \text { height }(\mathrm{cm})}{\mathrm{SCr}(\mathrm{mg} / \mathrm{dL})}$

Creatinine-based prediction models of eGFR for premature and low birth weight infants without kidney disease were introduced by Brion et al. in 1986 [13]. Brion, in collaboration with Schwartz, hypothesized that lower muscle mass in low birth weight and preterm infants would result in overestimation of eGFR based on the term infant equation. They evaluated $\mathrm{SCr}$ levels, $\mathrm{UCr}$ levels, and the plasma disappearance curve of inulin in 118 preterm and 84 term infants. The authors concluded that in premature and low birth weight infants, a lower constant of 0.33 should be used in estimations of GFR as it more accurately takes into account for their lower muscle mass:

eGFR $\mathrm{ml} / \mathrm{min} / \mathrm{m}^{2}=\frac{0.33 \times \text { height }(\mathrm{cm})}{\mathrm{SCr}(\mathrm{mg} / \mathrm{dL})}$

In 2009, using 349 children aged 1-16 years old who were enrolled in the chronic kidney disease in children (CKid) study, Schwartz et al. developed a simplified "bedside" GFR calculation utilizing a universal constant of 0.413 [14]:

$\mathrm{eGFR} \mathrm{ml} / \mathrm{min} / \mathrm{m}^{2}=\frac{0.413 \times \text { height }(\mathrm{cm})}{\mathrm{SCr}(\mathrm{mg} / \mathrm{dL})}$

This prediction model was subsequently validated by Staples et al. in a cohort of 573 predominantly healthy children, between 1 and 16 years of age, without kidney disease [15].

As $\mathrm{SCr}$ varies in children with cachexia and/or CKD, models employing serum cystatin-C (CysC), a cysteine proteinase produced by nucleated cells, as a biomarker of clearance have also been proposed in children. In a cohort of CKD and transplant patients, Zappitelli et al. proposed an estimation that incorporated serum CysC into a single model with height and $\mathrm{SCr}[16]$ :

eGFR $\mathrm{ml} / \mathrm{min} / \mathrm{m}^{2}$

$$
=\left(43.82 \times \mathrm{e}^{0.003 \times \mathrm{Ht}}\right) /(\mathrm{CysC} \cdot 0.635 \times \mathrm{SCr} \cdot 0.547)
$$

Abitbol et al. in 2014 found an agreement between GFR extrapolated from six inulin clearance studies and GFR calculated based on Zapitelli's combined equation in preterm and term neonates [17]. The study examined serum CysC levels and $\mathrm{SCr}$ levels within the first week of life for 60 preterm infants with gestational age (GA) $25-<37$ weeks and 40 term infants. All serum values were obtained $>48 \mathrm{~h}$ from birth. Creatinine-based estimates of GFR using the bedside Schwartz equation underestimated clearance in the cohort by over $20 \%$. The study concluded that $\mathrm{CysC}$ is a superior biomarker of clearance to $\mathrm{SCr}$ and improves the accuracy of noninvasive modeling of term and preterm neonatal glomerular filtration rates.

\section{Results of the Wilhelm-Bals et al.'s study}

In this issue of Pediatric Nephrology, Wilhelm-Bals et al. conducted a single-center, prospective study in newborns from Switzerland comparing clearance measured by singleinjection inulin and a new prediction model including weight and creatinine in term and preterm neonates [18]. Those with birth weight $<800 \mathrm{~g}$, hemodynamic instability, and severe anemia were excluded. The group compared their prediction model with the Brion et al., Zapitelli, and combined Zapitelli models [16]. Forty-eight infants were included in the study and inulin clearances were measured during days 1-6 of life in 44 patients ( 41 preterm neonates and 3 term neonates). The authors initially considered candidate variables of height, GA, weight, creatinine, and cystatin C. All variables except for cystatin $\mathrm{C}$ were found to be significantly correlated with inulin clearance on bivariate analysis. Multivariate linear regression was utilized to develop a parsimonious prediction model. Cystatin C, GA, log (creatinine), and weight were considered potential predictors. GA and cystatin $\mathrm{C}$ were ultimately removed from the model as they were not found to be significant predictors of GFR. The final model after correcting for the logarithmic transformation of serum creatinine was:

Predicted eGFR $\mathrm{ml} / \mathrm{min} / \mathrm{m}^{2}$

$$
=2.32 \times(\text { weight }(\mathrm{g}))^{0.64} /(\text { creatinine }(\mu \mathrm{mol} / \mathrm{L}))^{0.62}
$$

This model gave a mean prediction error of $-0.8 \mathrm{ml} / \mathrm{min} /$ $1.73 \mathrm{~m}^{2}(95 \% \mathrm{CI}-3.0-1.4)$, performing slightly better in this population than the neonatal Schwartz equation, which gave a mean prediction error of $2 \mathrm{ml} / \mathrm{min} / 1.72 \mathrm{~m}^{2}$ (95\% CI - 0.62.6). The mean prediction error was much higher for the Zappitelli and combined Zappitelli equations, which were $28.5 \mathrm{ml} / \mathrm{min} / 1.72 \mathrm{~m}^{2}$ (95\% CI 24.6-232.3) and $28.3 \mathrm{ml} / \mathrm{min} /$ $1.72 \mathrm{~m}^{2}$ (95\% CI 24.9-31.7), respectively.

\section{Significance and generalizability}

While this study is the first to utilize weight as a predictor of GFR in a cohort of predominantly preterm neonates during the first week of life, there are significant limitations before it will be accepted as a valid technique. The proposed eGFR model highlights the unique relationship between preterm and term neonates' eGFR and gestational age, height, and cystatin C. The significance of infant weight in the model may reflect the 
strong correlation between body weight and nephron number, thus affecting GFR. Although it is not clearly stated by the authors, it could be speculated that weight and GA were collinear variables within the model and thus, both were not included, which has been seen in other models [17].

One limitation of the use of weight as a model variable is that the authors do not specify whether the birth weight or the weight at the time of serum creatinine determination should be used in the equation. Term infants lose up to $10 \%$ of birth weight within the first week of life, with even greater losses in preterm infants. Utilizing weights other than birth weight could substantially affect GFR estimates and further clarification should be provided in future studies.

Infant height, a predictor in the majority of eGFR formulas, was not included in the initial full model even though it was significantly correlated to eGFR. Although the authors explained that they had a small sample size, the rationale for not including length in the model is not fully explained. Leger et al. developed a pharmacokinetic population model based on serum creatinine levels to estimate GFR utilizing weight and height in a cohort of children 8 months -18 years of age [19]. Further studies should assess whether height improves the prediction of GFR. This is important, as length has long been used in the signature formulas to estimate childhood GFR.

Another important limitation of this study is that the use of single-injection inulin clearance to directly measure GFR has not been validated in a large sample of premature infants. Urinary inulin clearance studies in neonates using inulin infusion were first described in 1975 by Guignard et al. [7]. These studies demonstrated a rapid increase in GFR in the first month of life, and a doubling of the GFR in the first 15 days of life. Coulthard showed that the continuous infusion of inulin method without urine collection gave a relatable result only when inulin was constantly infused for $24 \mathrm{~h}$ [20]. This finding was ascribed to the slow rate of inulin diffusion into the extracellular space of neonates. Using the 24-h inulin infusion method as a reference value, the same author found that the single-injection method correlated poorly with GFR when the single-injection clearance was estimated over $2 \mathrm{~h}$. Similarly, in 1979 , Fawer et al. showed that the single-injection inulin technique overestimated the urinary clearance of inulin by up to $30 \%$ in the first week of life [21].

The study also did not find cystatin $\mathrm{C}$ to be a significant predictor of GFR, which is discrepant from prior reports in the literature. As mentioned previously, Abitbol et al. demonstrated cystatin $\mathrm{C}$ to be a superior biomarker of clearance as compared with serum creatinine. Another study, also from Sweden, found no significant relationship between gentamicin clearance and measured cystatin $\mathrm{C}$ (the same study also did not find a strong relationship with creatinine) [22]. The debate as to whether cystatin $\mathrm{C}$ crosses the placenta is still, to some extent, unresolved. Contrary to what had been postulated previously, cystatin $\mathrm{C}$ may cross the placenta, but to a smaller extent than serum creatinine. One could suggest that Abitbol only looked at values after 48 days of life, while this paper looked at those on the first postnatal day, which may account for discrepancy of the findings. Alternatively, as suggested by the authors, the lack of significant correlation may be due to the small sample size. Regardless, the low intra-individual variability of cystatin $\mathrm{C}$ measurements in this study further lends support to use of cystatin $\mathrm{C}$ to detect AKI in this population.

As the cohort studied did not include extremely low birth weight infants, infants with AKI, or those with any hemodynamic instability and only 3 term neonates were included, the equation reflects GFR measured in a relatively healthy preterm cohort. This calculation can only be used within the first few days of postnatal life. Additionally, as all participants were of European descent, the model becomes less generalizable to a diverse worldwide population. This is of particular significance considering that African Americans have a higher risk of being born preterm and carrying the $A P O L 1$ risk allele [23].

\section{Conclusion}

The new model proposed by Wilhelm-Bals et al. offers a novel approach to predict GFR in preterm infants that needs validation. The study highlights the need to conduct studies and derive specific population-based calculations of eGFR as neonatal renal physiology is very different than infants older than 1 month of age. Further validation in a larger sample of ethnically diverse patients is needed to reexamine the relationship of GFR and cystatin $\mathrm{C}$ in this population, in addition to considering the inclusion of height in the model. Hopefully, future work will lead to more understanding of how to best assess GFR in preterm infants.

\section{References}

1. Filler G (2015) A step forward towards accurately assessing glomerular filtration rate in newborns. Pediatr Nephrol 30:1209-1212

2. Pottel $\mathrm{H}$ (2017) Measuring and estimating glomerular filtration rate in children. Pediatr Nephrol 32:249-263

3. Muhari-Stark E, Burckart GJ (2018) Glomerular filtration rate estimation formulas for pediatric and neonatal use. J Pediatr Pharmacol Ther 23:424-431

4. Jetton JG, Boohaker LJ, Sethi SK, Wazir S, Rohatgi S, Soranno DE, Chishti AS, Woroniecki R, Mammen C, Swanson JR, Sridhar S, Wong CS, Kupferman JC, Griffin RL, Askenazi DJ, Neonatal Kidney Collaborative (NKC) (2017) Incidence and outcomes of neonatal acute kidney injury (AWAKEN): a multicentre, multinational, observational cohort study. Lancet Child Adolesc Health 1: 184-194 
5. Abitbol CL, DeFreitas MJ, Strauss J (2016) Assessment of kidney function in preterm infants: lifelong implications. Pediatr Nephrol 31:2213-2222

6. Kaskel FJ, Kumar AM, Lockhart EA, Evan A, Spitzer A (1987) Factors affecting proximal tubular reabsorption during development. Am J Phys 252:F188-F197

7. Guignard JP, Torrado A, Da Cunha O, Gautier E (1975) Glomerular filtration rate in the first three weeks of life. J Pediatr 87:268-272

8. Fawer CL, Torrado A, Guignard JP (1979) Maturation of renal function in full-term and premature neonates. Helv Paediatr Acta $34: 11-21$

9. Guignard JP, John EG (1986) Renal function in the tiny, premature infant. Clin Perinatol 13:377-401

10. Vieux R, Hascoet JM, Merdariu D, Fresson J, Guillemin F (2010) Glomerular filtration rate reference values in very preterm infants. Pediatrics 125:e1186-e1192

11. Schwartz GJ, Haycock GB, Edelmann CM Jr, Spitzer A (1976) A simple estimate of glomerular filtration rate in children derived from body length and plasma creatinine. Pediatrics 58:259-263

12. Schwartz GJ, Feld LG, Langford DJ (1984) A simple estimate of glomerular filtration rate in full-term infants during the first year of life. J Pediatr 104:849-854

13. Brion LP, Fleischman AR, McCarton C, Schwartz GJ (1986) A simple estimate of glomerular filtration rate in low birth weight infants during the first year of life: noninvasive assessment of body composition and growth. J Pediatr 109:698-707

14. Schwartz GJ, Munoz A, Schneider MF, Mak RH, Kaskel F, Warady BA, Furth SL (2009) New equations to estimate GFR in children with CKD. J Am Soc Nephrol 20:629-637

15. Staples A, LeBlond R, Watkins S, Wong C, Brandt J (2010) Validation of the revised Schwartz estimating equation in a predominantly non-CKD population. Pediatr Nephrol 25:2321-2326
16. Zappitelli M, Parvex P, Joseph L, Paradis G, Grey V, Lau S, Bell L (2006) Derivation and validation of cystatin C-based prediction equations for GFR in children. Am J Kidney Dis 48:221-230

17. Abitbol CL, Seeherunvong W, Galarza MG, Katsoufis C, Francoeur D, Defreitas M, Edwards-Richards A, Master Sankar Raj V, Chandar J, Duara S, Yasin S, Zilleruelo G (2014) Neonatal kidney size and function in preterm infants: what is a true estimate of glomerular filtration rate? J Pediatr 164:1026-1031 e1022

18. Wilhelm-Bals A, Combescure C, Chehade H, Daali Y, Parvex P (2019) Variables of interest to predict glomerular filtration rate in preterm newborns in the first days of life. Pediatr Nephrol. https:// doi.org/10.1007/s00467-019-04257-z

19. Leger F, Bouissou F, Coulais Y, Tafani M, Chatelut E (2002) Estimation of glomerular filtration rate in children. Pediatr Nephrol 17:903-907

20. Coulthard MG (1983) Comparison of methods of measuring renal function in preterm babies using inulin. J Pediatr 102:923-930

21. Fawer CL, Torrado A, Guignard JP (1979) Single injection clearance in the neonate. Biol Neonate 35:321-324

22. Nielsen EI, Sandstrom M, Honore PH, Ewald U, Friberg LE (2009) Developmental pharmacokinetics of gentamicin in preterm and term neonates: population modelling of a prospective study. Clin Pharmacokinet 48:253-263

23. Friedman DJ, Pollak MR (2016) Apolipoprotein L1 and kidney disease in African Americans. Trends Endocrinol Metab 27:204215

Publisher's note Springer Nature remains neutral with regard to jurisdictional claims in published maps and institutional affiliations. 